Bernhard Grümme ${ }^{1}$

Ruhr-Universität Bochum

\title{
„Die Jugend im Religionsunterricht ist auch nicht mehr die, die sie mal war": Der Pluralismusbegriff der Religionspädagogik und seine Tragfähigkeit angesichts neuerer Jugendforschungen
}

„In Deutschland hat jedes Kind neun bis dreizehn Jahre Religionsunterricht. Wieso dann gar so wenig hängen bleibt, um es mal so auszudrücken, ist unbegreiflich. Hier müssen die Bischöfe in der Tat ernsthaft darüber nachdenken, wie der Katechese ein neues Herz, ein neues Gesicht gegeben werden kann". ${ }^{2}$ Wer hier Klage führt über die angebliche Wirkungslosigkeit des Religionsunterrichts ist kein Geringerer als der inzwischen emeritierte Papst Benedikt. Der Religionsunterricht, so

1 Bernhard Grümme, Prof. Dr. theol.: Professor für Religionspädagogik und Katechetik an der KatholischTheologischen Fakultät der Ruhr-Universität Bochum. Studium der Geschichte, Katholischen Theologie und Erziehungswissenschaften, Referendariat, sechsjährige Tätigkeit als Studienrat am Märkischen Gymnasium Hamm, von 2004-2013 Professor für Katholische Theologie/Religionspädagogik an der PH Ludwigsburg, seit 1.4.2013 an der RuhrUniversität Bochum; Forschungsschwerpunkte: Grundlagentheorie der Religionspädagogik, Politische Dimensionen religiösen Lernens, religiöse Bildungstheorien, religionspädagogische Anthropologie. E-mail: Bernhard.Gruemme@rub.de.

2 Benedikt XVI., Licht der Welt. Der Papst, die Kirche und die Zeichen der Zeit. Ein Gespräch mit Peter Seewald, Freiburg i. Br. 2010, S. 169. 
sein Lamento, schaffe es nicht mehr, jenes Glaubenswissen zu verbreiten, das für eine gelungene christliche Existenz erforderlich sei. Weil der Religionsunterricht nicht mehr hinreichend die Tradition und das Evangelium verbreite, so der Papst, sei das Wissen nicht mehr vorhanden, aus denen Menschen ihren christlichen Glauben leben könnten. Die Konsequenz ist klar: Nicht nur der Zeitgeist, auch der Religionsunterricht und die Katechese seien für den Verlust an Glauben sowie an der Bereitschaft zur Partizipation an institutionellen Praktiken wie Gottesdienstbesuch und kirchlichen Riten mitverantwortlich. Was kann in dieser schwierigen Lage helfen? Nicht ein stärkeres Eingehen auf die Verstehensleistungen und Verständnisbedingungen der Jugendlichen. Denn es könnte ja sein, dass diese gar nicht mehr verstehen, gar nicht mehr affektiv, kognitiv und volitiv nachvollziehen können, welches befreiende Potential in der christlichen Botschaft liegt. Nein, offensichtlich hilft hier nur eine verstärkte Hinwendung zu einer elementarisierten Verkündigung zentraler Inhalte des Glaubens, wie der Papst als damaliger Kardinal Ratzinger bereits in seiner berühmt-berüchtigten Rede in Frankreich zur Lage der Katechese im Jahr 1983 ausgeführt hatte. ${ }^{3}$

Drei Beobachtungen stechen ins Auge:

1. Man mag sich angesichts der verwickelten Debatten der Lernpsychologie darüber wundern, wie wenig Papst Benedikt die Forschung rezipiert, nach der die Subjekte nicht wie leere Gefäße die Inhalte einfach aufnehmen, die ihnen vermittelt wurden. Der Lehr-Lern-Kurzschluss ist längst widerlegt und hat einer ausdifferenzierten Dialektik von Vermittlungsund Aneignungsdidaktik Platz gemacht. ${ }^{4}$ Die Subjekte lernen nur, wenn sie sich die Lerngegenstände nach eigenen Gesetzen anverwandeln.

2. Man mag sich darüberhinaus darüber wundern, wie sehr Papst Benedikt Katechese und Religionsunterricht in einem Atemzug nennt und scheinbar keine Differenzierungen vornimmt. Dabei liegt doch eine der großen Errungenschaften neuerer Religionspädagogik und Katechese genau darin, beide getrennt zu halten. Katechese in der Gemeinde ver-

3 J. Ratzinger, Die Krise der Katechese und ihre Überwindung. Rede in Frankreich, Einsiedeln 1983.

4 B. Porzelt, Grundlegung religiöses Lernen, Stuttgart 2009. 
sammelt diejenigen, die sich bereits für den Glauben entschieden haben und darin Begleitung wünschen. Religionsunterricht in der Schule jedoch betrifft alle, auch diejenigen, die religiös gleichgültig oder sich gar gegen den Glauben entschieden haben. Katechese zielt auf Glaubensvertiefung, Religionsunterricht auf erfahrungsbegründete Bildung und kritische Urteilsbildung über den Glauben. ${ }^{5}$ Wenn man die Jugendlichen in ihrer Freiheit und Katechese wie den Religionsunterricht in ihrem Dienst an der Mündigkeit und an der Glaubensentwicklung der Subjekte ernstnimmt, dann muss man an dieser Trennung festhalten.

Papst Benedikt scheint demnach einem lernpsychologischen und katechetischen Missverständnis des Religionsunterrichts zu unterliegen.

3. Es ist aber zugleich höchst bemerkenswert, dass Papst Benedikt sich in seiner Diagnose mit Ergebnissen neuerer religionspädagogischer Forschungen zum Religionsunterricht trifft. Dort wurde etwa von einer Forschergruppe um den einflussreichen Religionspädagogen Rudolf Englert herausgearbeitet, dass nach 13 oder 12 Jahren Religionsunterricht ziemlich wenig Glaubenswissen herauskommt. Der Erkenntnisfortschritt entspreche nicht dem großen Aufwand, der religionsdidaktisch betrieben werde. Insgesamt gesehen sei in einem bedrückenden Ausmaß eine erhebliche „Wirkungsschwäche des Religionsunterrichts“ zu konstatieren. ${ }^{6}$ Für unser Erkenntnisinteresse ist die Begründung höchst aussagekräftig. Der Religionsunterricht habe sich hohe Verdienste um die Subjektorientierung erworben. Die Unterrichtsforschung hat einen sehr starken Einbezug der Schülerinnen und Schüler bei der Themenfindung, bei der Dramaturgie und der Kommunikation im Unterricht nachgewiesen. ${ }^{7}$ Nein, das Problem sei trotz Defiziten in der Berücksichtigung der Pluralität heutiger Lebenswelten, in denen sich die Jugendlichen bewegen, weniger die Orientierung an den Schülern. Es sei gerade deren Unterforderung und zu geringe Aktivierung durch herausfordernde kog-

\footnotetext{
B. Grümme, Öffentliche Religionspädagogik. Religiöse Bildung in pluralen Lebenswelten, Stuttgart 2015.

${ }_{6}^{6}$ R. Englert, Religion gibt zu denken. Eine Religionsdidaktik in 19 Lehrstücken, München 2013, S. 24.

7 R. Englert u.a., Innenansichten des Religionsunterrichts. Fallbeispiele, Analysen, Konsequenzen, München 2014, S. 220f.
} 
nitive Impulse. Man habe den Mut zu einer Instruktion verloren, wie dies durch Lehrerstudien von John Hattie ja eindrucksvoll bestätigt wurde. Dass Religion zu denken gibt, dies würde als didaktisches Grundaxiom des Religionsunterrichts zu wenig realisiert. ${ }^{8}$

Ähnlich wie im Urteil des Papstes wird hier zwar ein erhebliches Defizit des Religionsunterrichts konstatiert, aber anders als dort geht dies nicht zu Lasten der Anerkennung der kognitiven und religiösen Eigenwirklichkeit der Jugendlichen. Wenn man Religionsunterricht und Katechese planen, gestalten, wenn man sie denken will, dann kommt man nicht umhin, die Jugendlichen selber in den Blick zu nehmen, deren Lebenswelten, deren Vorstellungen, deren Sehnsüchte, deren Hoffnungen und Orientierungslosigkeiten zu untersuchen. Tut man dies nicht, dann geraten Katechese und Religionsunterricht zur fremdbestimmenden Indoktrination. Der Jugendkatechismus YOUCAT ist ein bedrückendes Beispiel hierfür. Und man wird gespannt sein, inwieweit die von Papst Franziskus angeregte Jugendbibel stärker aneignungsorientiert sein wird.

Auch die katholischen Bischöfe in Deutschland nehmen übrigens die Jugendlichen anerkennend in den Blick, wenn sie in ihrem Schreiben zum Religionsunterricht die veränderten Bedingungen des Aufwachsens und der Pluralisierung von Religion in der Spätmoderne als Voraussetzung für den Religionsunterricht anerkennen. Da ist von Traditionsabbruch die Rede, davon, dass elementare Kenntnisse religiöser Praktiken wie das Vaterunser und Symbole wie das Kreuzzeichen nicht mehr vorhanden seien. Diese Bedingungen gelte es ernstzunehmen, nicht durch ungebrochene Vermittlungsversuche feststehender Glaubensinhalte zu überformen. Dies liefe dem diakonischen Auftrag des Religionsunterrichts zuwider, der eben an den Jugendlichen selber interessiert sei und ihnen ein gelingendes Leben ermöglichen wolle. ${ }^{9}$

Wenn auch in unterschiedlicher Intensität sehen sowohl die katholischen Bischöfe als auch einflussreiche Religionspädagogen in der Pluralisierung der Lebenswelten und der Religion einen starken Faktor

8 R. Englert, Religion gibt zu denken..., op. cit., S. 15-100.

9 Der Religionsunterricht vor neuen Herausforderungen, Hg. Der Deutschen Bischofkonferenz (Die deutschen Bischöfe 80), Bonn 2005. 
dafür, dass Katechese und Religionsunterricht neu gedacht werden müssen. Andernfalls würden sie wirkungslos wie fremdbestimmend. Gegenwärtige Religionspädagogik spricht von einer zu gewinnenden Pluralitätsfähigkeit als Paradigma. Doch reicht eine solche Diagnose und normative Zielbestimmung aus? Müssten nicht über vor allem religiöse Vielfalt weitere Prozesse der Ausdifferenzierung berücksichtigt werden? Könnte es nicht sein, dass der Religionsunterricht auch daran scheitert, weil er die sozialen Bedingungen und die damit zusammenhängenden Sprachkompetenzen zu wenig beachtet? Müsste nicht die Konzentration auf Pluralität einer stärkeren Sensibilität für Heterogenität weichen, in der neben religiöser Vielfalt auch soziale und kulturelle Differenzen gedacht werden? Dieses Votum für Heterogenität als Paradigma der Religionspädagogik ist jedenfalls meine These, die ich im Folgenden in drei Schritten erläutern und begründen will.

Erstens will ich den Pluralitätsbegriff erläutern. Zweitens will ich dessen religionspädagogische Brisanz anhand aktueller religionssoziologisches Studien zur Religiosität Jugendlicher erhärten und schließlich drittens dann den Mehrwert des Heterogenitätsbegriffs markieren.

\section{Pluralitätsfähigkeit als Paradigma der Religionspädagogik}

Spätestens mit Blick in eine Schulklasse wird deutlich, dass man die Wirklichkeit nicht mehr als Einheit begreifen kann. Alles hat sich vervielfältigt, ist plural geworden. Der Plural, nicht der Singular regiert. Dies kann man in, hier nur exemplarisch für die Vervielfältigung des Ganzen insgesamt stehenden Aspekten wie Religion, Weltanschauung, Kultur, Gesellschaft, Wissenschaft, Politik bis in die lebensweltlichen Praktiken und in die pädagogischen Handlungszusammenhänge hinein beobachten.

Insbesondere die religiöse Pluralisierung ist unübersehbar, die sowohl innerhalb der Religionen stattfindet wie als Vervielfältigung des Religiösen selber sich ereignet. ${ }^{10}$ Entgegen den Annahmen der

10 K. Gabriel, Pluralisierung und Individualisierung in Gesellschaft, Religion und Kirche, [in:] Christliche Identität in pluraler Gesellschaft, Hg. H. Münk, M. Durst, Freiburg-Schweiz 2005, S. 33. 
Säkularisierungstheorie führt die radikalisierte Moderne nämlich keineswegs zum Verschwinden von Religion. Stattdessen diagnostiziert man die „Wiederkehr der Götter"11, die „Rückkehr der Religionen“, ${ }^{12}$ eine ,postsäkulare Gesellschaft" . ${ }^{13}$ Parallel dazu macht sich ein zunehmend brisanter werdender Atheismus bemerkbar. Ein Prozess des Glaubensverlustes ist $\mathrm{zu}$ konstatieren, insofern die religiöse Zugehörigkeit zu religiösen Institutionen dramatisch abgenommen und die religiöse Praxis, die am regelmäßigen Gottesdienstbesuch gemessen wird, deutlich zurückgegangen ist. Dies wird auch nicht durch die punktuell feststellbare Vitalisierung von Religion in Erweckungsbewegungen und anderen nichtinstitutionellen Religionsformen ausgeglichen. ${ }^{14}$ Eher ließe sich der gegenwärtige Kontext als ,postreligiös und postsäkular“ charakterisieren, ${ }^{15}$ in dem sich die Religionen unter der Oberfläche der Vervielfältigung und des religiösen Aufbruchs auch qualitativ verändert haben. Religionen werden dispersiv. Tradierte Inhalte und Praxisformen werden individuell zerlegt, neu konstruiert, synkretistisch vermengt und in andere Formen und Formate bis hin zu einer subjektiven, „unsichtbaren Religion“ (Thomas Luckmann) überführt. ${ }^{16}$ Aus einer großen Transzendenz ist nun allerdings in dieser pluralisierten Religion nicht selten eine kleine Transzendenz geworden, eine Religion ohne Gott, die kosmischer Frömmigkeit und privatisierten Erlebnissen mehr Plausibilität abgewinnt. ${ }^{17}$

Insgesamt gesehen stellt sich religionsphänomenologisch das Problem der Religion im Kontext der Spät- oder Nachmoderne auf mehreren Ebenen

11 F. W. Graf, Die Wiederkehr der Götter. Religion in der modernen Kultur, München 2004.

12 M. Riesebrodt, Die Rückkehr der Religionen: Fundamentalismusund der „Kampf der Kulturen“, München 2000.

13 J. Habermas, Zwischen Naturalismus und Religion. Philosophische Aufsätze, Frankfurt a. M. 2005, S. 116.

14 D. Pollack, Religionund Moderne. Versuch einer Bestimmung ihres Verhältnisses, [in:] Gottesrede in postsäkularer Kultur, Hg. P. Walter, Essen 2007, S. 43.

15 H. J. Höhn, Postsäkular. Gesellschaft im Umbruch - Religion im Wandel, Paderborn 2007, S. 25 .

H. J. Höhn, Postsäkular..., op. cit., S. 34.

17 W. Ritter, Leid und Gott - didaktisch reflektiert, "Religionspädagogische Beiträge“ 59 (2007), S. 3ff; R. Englert, Gottesglaube hier und heute. Empirische Erkundung und theologische Herausforderung, „Theologische Revue“ 103 (2007) Nr. 3, S. 182 ff. 
dar. Drei Ebenen können wir dabei unterscheiden: eine Makroebene, eine Mesoebene, eine Mikroebene.

Auf der Makroebene gesellschaftlich-kultureller Systeme ist Religion unter Verlust ihrer universalen Orientierungsfunktion zu einem Subsystem unter anderen geworden. Sie hat ihre integrierende Funktion für Gesellschaft und Kultur verloren und steht nun im Wettstreit mit anderen Weltanschauungen und Ideologien um eine angemessene Interpretation der Wirklichkeit. Das Zeitalter der Vorherrschaft einer christlichen Interpretation von Kultur und Gesellschaft ist vorbei.

Auf der Mesoebene wird die Kirche als Institution vor allem nach ihrer gesellschaftlichen Funktion, nicht aber nach ihrem eigenen Anspruch bemessen. Zwar ist sie in der Bundesrepublik Deutschland nach wie vor im Bildungsbereich, im sozial-caritativen Bereich oder im Betreuungsbereich unverzichtbar. Man denke an kirchliche Schulen, an kirchliche Kindergärten und kirchliche Krankenhäuser. Jedoch wird sie in ihrem Dienst für die Gesellschaft gewichtet.

Auf der Mikroebene wird die Religion eingespielt in die Pluralisierung der Identität des Individuums mit diversifizierenden Teilidentitäten und Rollenzuschreibungen..$^{18}$ Die Religionspädagogik soll dies nicht nur wahrnehmen. Sie soll in ein reflektiertes, kritisches Verhältnis dazu treten. In diesem Sinne will Religionspädagogik spätestens seit Beginn des 21. Jahrhunderts pluralitätsfähig sein. ,Pluralitätsfähigkeit kann in einer ersten Annäherung als reflektierter Umgang mit Vielfalt verstanden werden. Gedacht wird dabei an verschiedene Formen von Pluralität - in sozialer, politischer, kultureller, religiöser und weltanschaulicher Hinsicht" ${ }^{19}$ Nicht Einheitlichkeit, sondern Vielfalt, nicht Uniformität, sondern Differenz soll der kategoriale Rahmen ihrer praktischen wie theoretischen Selbstverständigungsprozesse sein.

Um aber gerade inmitten dieser Pluralität dennoch handlungs- und orientierungsfähig zu sein, braucht sie genau darin ein normatives

18 H. G. Ziebertz, Gesellschaftliche und jugendsoziologische Herausforderungen für die Religionsdidaktik, [in:] Religionsdidaktik. Ein Leitfaden für Studium, Ausbildung und Beruf, Hg. G. Hilger, München 2010, S. 76-105.

19 F. Schweitzer, Interreligiöse Bildung, Religiöse Vielfalt als religionspädagogische Herausforderung und Chance, Gütersloh 2014, S. 196. 
Grundgerüst ihres Sinns, ihrer Begriffsbildung und ihrer praktischen wie theoretischen Orientierungen. Demnach ist eine Religionspädagogik dann pluralitätsfähig, ,wenn sie Abschied von Uniformität nimmt und gleichzeitig die Kraft zu klärenden Unterscheidungen behält" “. ${ }^{20}$ Pluralitätsfähigkeit ist damit zum Leitbegriff der Religionspädagogik avanciert. Differenz soll wahrgenommen, beurteilt und kritisch-produktiv in der für Religionspädagogik eigentümlichen Theorie-Praxis-Dialektik bearbeitbar werden. Religionspädagogische Handlungsprozesse zielen demnach auf eine Wahrnehmungs-, Handlungs-, Sprach- und Urteilsfähigkeit der Jugendlichen. ${ }^{21}$ In dem Maße, wie sie sich am Pluralismusparadigma ausrichtet, räumt die Religionspädagogik ihrer Pluralitätsfähigkeit selber eine axiomatische, geradezu paradigmatische Bedeutung ein. Wie dringlich dies ist, zeigt ein Blick in neuere empirische Forschungen zur Religiosität Jugendlicher.

\section{Undogmatisch religiös? Jugendliche Religiositäten}

Jugendliche Religiosität ist inzwischen Gegenstand intensiver religionssoziologischer und religionspädagogischer Forschung. ${ }^{22}$ Es spricht für die Mehrheit der Katholischen Bischöfe in Deutschland, dass sich sich keinen Illusionen hingeben. So zeigen empirische Forschungen die Entkonfessionalisierung und Enttraditionalisierung in eklatanter Weise auf. Burkard Porzelt macht auf der Basis seiner qualitativ-empirischen Forschungen zu Intensiverfahrungen Jugendlicher auf die bleibende Disparität zwischen christlich-religiöser Sprache und der Sprache Jugendlicher aufmerksam: Zwischen jugendlichen Lebenserfahrungen

20 R. Englert u.a., Einleitung, [in:] Welche Religionspädagogik ist pluralitätsfähig? Kontroversen um einen Leitbegriff, Hg. R. Englert u.a., Freiburg i. Br. 2012, S. 11.

${ }_{21}$ H. G. Ziebertz, Grenzen des Säkularisierungstheorems, [in:] Entwurf einer pluralitätsfähigen Religionspädagogik, Hg. F. Schweitzer u.a., Gütersloh-Freiburg i. Br. 2002, S. 67.

22 Zwischen Religion und Religiosität. Ungebundene Religionskulturen in Religionsunterricht und kirchlicher Jugendarbeit - Erkundungen und Praxis, Hg. U. Kropac, U. Meier, K. König, Regensburg 2015. 
und christlichen Glaubenserfahrungen gibt es, so Porzelt, „keinerlei sprachliche Berührungspunkte" “. ${ }^{23}$

Diese Pluralisierung ist in verschiedenen Forschungsprojekten nachgewiesen worden ${ }^{24}$ Insgesamt wird die Pluralisierung innerhalb der christlichen Religion wie die Pluralisierung von Religion wie von Religiosität deutlich. Von der Vorstellung auszugehen, im Religionsunterricht säßen nur an Religion Interessierte oder gar dem Glauben zugewandte Jugendliche, dies greift zu kurz. Schon im konfessionellen Religionsunterricht, aber mit Abstrichen auch in der Katechese ist also eine Vielzahl ganz unterschiedlicher Religiositäten zu konstatieren. Die Religionspädagogik geht nun nicht hin, und misst die Jugendlichen an einem vordefinierten Begriff des Glaubens, der dann auf die Jugendlichen als Kategorie übertragen wird. Damit wird lediglich eine Defizithermeneutik vollzogen, die den Jugendlichen immer nur ihre eigene Dekadenz aufzeigt und nachweisen will, was ihnen in Hinsicht auf diesen Glaubensbegriff noch fehlt. Stattdessen würdigt sie, was die Jugendlichen bereits an religiösen Schätzen haben, kritisiert aber auch, inwieweit dort statt Gott bereits sich die Götzen des Kapitalismus oder anderer entfremdender Kräfte mit religiöser Überhöhung breitgemacht haben und wie in diese Lebenswelten, in diese bestimmten Formen sichtbarer und unsichtbarer Religion die tradierte jüdisch-christliche Tradition kritisch wie befreiend eingespielt werden kann, weil sie eben ein zentraler Beitrag zu einem gelingenden Leben in der Spätmoderne darstellt. ${ }^{25}$

Offensichtlich gibt es keine Alternative zum Pluralismusparadigma, das ja übrigens nicht nur für die Jugendlichen gilt. Auch die Lehrenden in Katechese und Schule stehen doch ihrerseits in diesem lebensweltlichen und kulturellen Rahmen. Nur scheint dieses Paradigma, wie oben

${ }^{23}$ B. Porzelt, Jugendliche Intensiverfahrungen. Qualitativ-empirischer Zugang und religionspädagogische Relevanz, Graz 1999, S. 257.

24 H. G. Ziebertz, Gesellschaftliche und jugendsoziologische..., op.cit., S. 101-102; G. Hilger, Heilig ist mir... Texte von Jugendlichen, „Katechetische Blätter“ 124 (1999), S. 411-412; M. Sellmann, Zuhören Austauschen Vorschlagen Entdeckungen pastoraltheologischer Milieuforschung, Würzburg 2012; Jugend 2015. Eine pragmatische Generation im Aufbruch, Hg. Shell Deutschland Holding, Hamburg 2015.

25 S. Pemsel-Maier, M. Schambeck, Keine Angst vor Inhalten! Systematisch-theologische Themen religionsdidaktisch erschließen, Freiburg i. Br. 2015. 
bereits von mir angedeutet, an seine Grenzen zu stoßen. Zumindest muss die Frage erlaubt sein, ob nicht einer der Gründe für die Probleme des Religionsunterrichts auch darin liegen, dass die Komplexität der gegenwärtigen Ausdifferenzierungsprozesse in der Spätmoderne nicht komplex genug begriffen werden. Darauf deuten Ergebnisse der Unterrichtsforschung hin.

\section{Religiöse Konstruktionen bildungsferner Jugendlicher}

Bislang hat man in der religionspädagogischen Erforschung der Religiosität Jugendlicher zu wenig gesehen, inwieweit sich die lebensweltlichen und sozialen Hintergründe in der Weise, Religiosität zu eben, sich über Religion zu äußern oder den eigenen Glauben zu vollziehen niederschlagen. Dabei hat die Bildungssoziologie eine enge Interdependenz von Bildung, Sprache und sozialer Schichtung nachweisen können. ${ }^{26}$ Für die Religionspädagogik stellt dies eine enorme Herausforderung dar. Es wird zumeist weder differenziert, inwiefern sich hier schulformspezifische Gegebenheiten niederschlagen, noch wird näher reflektiert, ob und ggf. inwiefern sich der Kontext der Schulen und die sozialen Lebenswelten und Strukturen der Schülerinnen und Schüler auf das Gottdenken selber auswirken. Unausgewiesen bleiben vor allem die Präsuppositionen, die stillschweigenden Voraussetzungen, die gemacht werden, die Annahmen an Kompetenzen, Fähigkeiten, Fertigkeiten und Motivationen auf Seiten der Jugendlichen. Es wird nicht hinreichend exploriert, ob die hier dokumentierten eindrucksvollen Leistungen der Jugendlichen eine generelle Leistung von Jugendlichen in diesem Alter sind oder eben aus einem bestimmten sozialen und kulturellen Setting hervorgehen. Es wird so getan, als wenn man diese verallgemeinern und universalisieren könnte. Doch ist dies wirklich generell der Fall? Gilt dies überall, für Jugendliche in Bayern wie im Ruhrgebiet, für Jugendliche in der Hauptschule oder der Realschule und Berufsschule, für Kinder von Lehrern, von Professoren wie für Kinder von Angestellten, Facharbeitern

26 B. Grümme, Bildungsgerechtigkeit. Eine religionspädagogische Herausforderung, Stuttgart 2014, S. 30-100. 
oder Hartz-IV-Empfängern? Neuere Religionssoziologie hat aufgezeigt, dass insbesondere bei gesellschaftlich und kulturellen Jugendlichen die Religiosität ein Distinktionsmarker ist, durch den sie sich gerade Identität und Selbstvergewisserung verschaffen, die ihnen gesellschaftlich oft nicht zugestanden wird. Indem sie sich eine Religiosität aneignen, indem sie sich in eine bestimmte Religion selber hinein sozialisieren, wird Religiosität wie Religion bewusst „strategisch eingesetzt “ ${ }^{27}$ Nach empirischen Studien von Dörthe Vieregge finden sich bei Jugendlichen in prekären Lebenslagen personale Gottesbilder, die Gott in Beziehungen verstrickt denken. Mehr noch: um einem Gefühl der nochmaligen Stigmatisierung und Exklusion zu begegnen, konnte Vieregge sogar ein Interesse der Jugendlichen an verfasster Religion und einem expliziten Gottesbekenntnis nachweisen. ${ }^{28}$

Diese bewusste Verwendung von Religion und Religiosität zur eigenen Identitätssicherung in den prekären Lebensverhältnissen der Spätmoderne wird in der Religionspädagogik bislang zu wenig berücksichtigt. So stellen sich die religiösen Aussagen von Jugendlichen aus bildungsfernen Schichten, wie sie vor allem in der Hauptschule zu finden sind, ganz unterschiedlich dar. Dies ist das Ergebnis erster Untersuchungen zum Sprachgebrauch religiöser Semantik bei Schülerinnen und Schülern diverser Schulformen. ${ }^{29}$ Frank Lütze konnte in breiten empirischen Studien einen signifikanten Zusammenhang zwischen „Bildung(sgang) und religiöser Orientierung“ nachweisen. ${ }^{30}$ Besonders markant sind folgende Ergebnisse:

1. Hauptschüler scheinen im Unterschied zu anderen Schülern besonders zurückhaltend bei der Zustimmung zu explizit christlichen Bekenntnissen zu sein.

2. Hauptschüler erleben ihr Leben im Unterschied zu Schülern in höheren formalen Bildungsgängen weniger durch Freiräume geprägt,

27 M. Sellmann, Jugendliche Religiosität als Sicherungs- und Distinktionsstrategie im sozialen Raum, [in:] Jugend, Religion, Religiosität. Resultate, Probleme und Perspektiven der aktuellen Religiositätsforschung, Hg. U. Kropac, U. Meier, K. König, Regensburg 2012, S. 46.

28 D. Vieregge, Religiosität in der Lebenswelt sozial benachteiligter Jugendlicher. Eine empirische Studie, Münster 2013, S. 240-244.

29 B. Grümme, S. Altmeyer, Gerechtigkeit durch religiöse Bildung, „Theologisch-Praktische Quartalsschrift“ 3 (2014) S. 314-324.

30 F. Michael Lütze, Religiöse Bildung im Hauptschulbildungsgang, „Theo-Web. Zeitschrift für Religionspädagogik“ 10 (2011), S. 62. 
sondern stärker als schicksalhaft vorgegeben. Dieser Fatalismus korreliert mit einem Gottesbild, das Gott als in den Weltlauf eingreifende und die Welt geradezu lenkende Macht konzeptualisiert. Damit sind den Aspekten der Weltentstehung, der Weltlenkung, des Schöpfungs- und des Vorsehungsglaubens ein besonderes Gewicht im Denken der Hauptschüler gegeben. Weniger dialogische Beziehungen zu Gott als ein eher passives Bezogensein stehen im Vordergrund.

3. Dieses Gefühl, im Leben ohnehin nichts Wesentliches ändern zu können, korrespondiert eine vergleichsweise ,größere Affinität zu mantischen und okkulten Praktiken “. ${ }^{31}$ Die starke Nähe zu konkreten, anschaulichen, mythisch-wortwörtlichen Strukturen ihrer religiösen Rede verdeutlicht zumindest einen erkennbaren Zusammenhang zwischen religiöser Entwicklung und Bildungsgang.

Die Konsequenzen einer solch schonungslosen Analyse für die Religionspädagogik liegen vor allem auf zwei Ebenen:

Erstens werfen die höchst prekären, von Armut, Bildungsungleichheit und drohender Arbeitslosigkeit bedrohten Lebenslagen von Hauptschülern "gewichtige materialethische Fragen auf (etwa nach Chancen- und Bildungsgerechtigkeit im Blick auf Menschen aus unterprivilegierten Verhältnissen), zu denen ein Religionslehrer bzw. eine Religionslehrerin im Hauptschulbereich in fachkundiger Weise gesprächsfähig sein sollte “" ${ }^{32}$

Zweitens fordert eine solche Analyse die Inhalte, die Didaktik und das Bildungsverständnis der Religionspädagogik radikal heraus. Die Zusammenhänge zwischen „Schulbildung, sozialen Lagen und religiösen Vorstellungen“ zeigen sich in der im Kontext prekärer Lebenslagen feststellbaren Verstärkung von Machtaspekten im Gottesbild. ${ }^{33}$ Sie stellen die Religionspädagogik vor folgende Fragen: Wie kann von Gott im RU geredet werden, der beziehungswillig und mit den Menschen in der Geschichte heilend, befreiend, vergebend unterwegs ist, wenn Gott vor allem als hereinbrechende, determinierende Macht verstan-

31 F. M. Lütze, Religionslehrer/in an Hauptschulen werden. Überlegungen zu schulformspezifischen Akzenten im theologischen Studium, "Theo-Web. Zeitschrift für Religionspädagogik“ 10 (2011), S. 88.

32 F. M. Lütze, Religionslehrer/in an Hauptschulen werden..., op. cit., S. 89.

33 F. M. Lütze, Religionslehrer/in an Hauptschulen werden..., op. cit., S. 89. 
den wird? Wie kann in einem lebensweltlichen Zusammenhang die Rechtfertigungsbotschaft zur Sprache gebracht werden? Die Kritik an menschlicher Leistungsorientierung und die Warnung vor menschlicher Hybris erhält im Lichte einer zu erwartenden Karriere von Arbeitslosigkeit und Geringbeschäftigung einen massiv „zynischen Beigeschmack“ und droht in billige Vertröstung umzuschlagen. ${ }^{34}$

Didaktisch erwächst aus den stärker handlungsorientierten Zugängen der Hauptschulpädagogik eine Gefahr. Insofern lebenspraktische, erfahrungs- wie erlebnisbezogene und handlungsorientierte Verfahren im RU der Hauptschule eine besonderes Gewicht erhalten, erscheint es unverzichtbar, den Schülern sowohl in elementarisierter Form exegetische Kenntnisse, Fertigkeiten und kritische Selbstreflexion als auch Möglichkeiten der reflexiven Distanzierung einzuräumen. Wird eine solche Balance zwischen Innenperspektive und reflektierender Distanzierung verfehlt, droht dies am Ende unversehens in eine katechetische Einweisung in das Christentum umzuschlagen..$^{35}$

Dies alles sind so gravierende Anfragen, dass sie den Rahmen des Pluralismusparadigmas sprengen.

\section{Heterogenitätsfähige Religionspädagogik. Resultat und Perspektiven}

Nach dem bisher Ausgeführten scheint das Pluralismusparadigma nicht hinreichend geeignet zu sein, die komplexen wie ausdifferenzierten religiösen, wie sozialen und kulturellen Lebensformen, Semantiken und Praxen Jugendlicher angemessen zu berücksichtigen. Bislang haben wir weitere Faktoren nicht einmal erwähnt, die hier zudem in Anschlag zu bringen wären und die zunehmend schulpädagogisch wie religionspädagogisch diskutiert werden: die Frage von Behinderung als Herausforderung für eine Inklusive Religionspädagogik, die Frage von

34 F. M. Lütze, Religionslehrer/in an Hauptschulen werden..., op. cit., S. 90.

35 F. M. Lütze, Religionsunterricht im Hauptschulbildungsgang. Konzeptionelle Grundlagen einer Religionsdidaktik für den Pflichtschulbereich der Sekundarstufe I, Leipzig 2011, S. 68-92. 
Gender und Queer als Herausforderung für eine geschlechtergerechte Religionspädagogik, die Frage von Ungleichheit als Herausforderung für eine bildungsgerechte Religionspädagogik. Diese Ausdifferenzierung übersteigt das begriffliche Gefüge des Pluralitätsbegriffs. Wäre hier nicht der Heterogenitätsbegriff weiterführend? In der pädagogischen Heterogenitätsforschung wird unter Heterogenität so Unterschiedliches verstanden wie „kognitive Leistungsfähigkeit (Intelligenz, fachliche Leistung, aber auch Lernbehinderung), soziale Herkunft (Sozialschicht, Familienstruktur, Migrationshintergrund, religiöse Einbindung etc.), die Geschlechtszugehörigkeit und das ,Alter'“. ${ }^{36}$ Das Potential eines solchen Begriffs liegt auf der Hand: Er ist deutlich komplexer, vielschichtiger und differenzierter als der Pluralitätsbegriff. Er macht die Divergenz unterschiedlicher Funktionssysteme, Rollen und Lebenswelten trennscharf, in den sich die Jugendlichen bewegen. Gerade also in ihrer Orientierung an der Würde und Autonomie der Jugendlichen als Subjekte religiöser Bildung hätte die Religionspädagogik heterogenitätsfähig zu werden im Sine ihrer eigenen Wahrnehmungs-, Urteils-, Sprach- und Handlungsfähigkeit. Im Rahmen ihrer spezifischen Bedingungen müsste dies auf ihre Weise auch für die Katechese gelten. Zugleich aber bleiben offene Fragen, die geklärt werden müssen, soll die Religionspädagogik sich paradigmatisch umorientieren.

1. Wie ist das Verhältnis zwischen dem Pluralismus- und dem Heterogenitätsparadigma? Bislang hat man eher den Eindruck einer unreflektierten semantischen Verschiebung, die die semantischen und grundlagentheoretischen Konsequenzen nicht hinlänglich beachtet. ${ }^{37}$

2. Kann der Heterogenitätsbegriff die Wechselwirkungen zwischen den einzelnen Momenten der Heterogenität angemessen würdigen? Die gegenseitige Verstärkung von sozialer Lage, Religion und kulturellem Hintergrund, die ja durch die Flüchtlingsfrage nochmals enorme Dynamik erfährt, sind inzwischen als Faktoren für de Unterricht

36 M. Trautmann, B. Wischer, Heterogenität in der Schule, Eine kritische Einführung, Wiesbaden 2011, S. 40.

37 C. Gärtner, Religionsunterricht - ein Auslaufmodell? Begründungen und Grundlagen religiöser Bildung in der Schule, Paderborn 2015, S. 14, 99. 
nachgewiesen. Bietet hier nicht der Intersektionalitätsbegriff bessere Möglichkeiten? ${ }^{38}$

3. Müsste nicht noch stärker in einer komparativen Religionspädagogik untersucht werden, inwieweit sich die Lebenswelten Jugendlicher in unterschiedlichen Ländern auf religiöses Lernen je unterschiedlich auswirken? Gibt es hier in den Transformationsprozessen der Spätmoderne auf verschiedenen Ebenen Ungleichzeitigkeiten, nachholende Prozesse, Gegenläufigkeiten, so dass man vielleicht sogar voneinander lernen kann? Ein Vergleich zwischen Deutschland und Polen wäre hier herausfordernd wie ertragreich.

All dies, und so komme ich zum Schluß, würde vielleicht dazu beitragen, anders als der Papst nicht allzu pessimistisch auf religiöse Bildungsprozesse zu schauen und den Jugendlichen auch in Fragen von Religion und Glauben einfach mehr zuzutrauen.

38 Katharina Walgenbach, Heterogenität - Intersektionalität - Diversity in der Erziehungswissenschaft, Opladen Toronto 2014. 


\section{Summary}

"Die Jugend im Religionsunterricht ist auch nicht mehr die, die sie mal war": Der Pluralismusbegriff der Religionspädagogik und seine Tragfähigkeit angesichts neuerer Jugendforschungen

Der RU in der öffentliche Schule wird vorgeworfen, zu wenig religiöses Wissen zu vermitteln. Diesem Vorwurf kann angemessen nur begegnet werden, wenn die Pluralität verschiedener Zugänge der Jugendlichen zur Religion und zum Glauben berücksichtigt wird. Weil aber neben religiösen und kulturellen Aspekten auch gesellschaftliche und soziale Momente wichtig sind, wäre das Pluralismusparadigma der Religionspädagogik weiterzuentwickeln zu einem Heterogenitätsparadigma.

Schlüsselwörter: Pluralität, Heterogenität, Religiöse Bildung

"Youth in religious education isn't still the same".

The Paradigm of pluralism in religious education and its validity

in the perspective of empirical youth studies

Religious education as a subject in school is increasingly criticized for neglecting its duty to impart religious knowledge to the youngsters. Religious education can only deal with this critic by referring to the increasing pluralization in religion and belief. But due to the importance of social and economical structures beside cultural and religious aspects religious education might develop a paradigm of heterogeneity.

Keywords: heterogeneity, pluralism, religious education

\section{Bibliografie}

Benedikt XVI., Licht der Welt. Der Papst, die Kirche und die Zeichen der Zeit. Ein Gespräch mit Peter Seewald, Freiburg i. Br. 2010.

Der Religionsunterricht vorneuen Herausforderungen Hg. Der Deutschen Bischofkonferenz (Die deutschen Bischöfe 80), Bonn 2005.

Englert R., Gottesglaube hier und heute. Empirische Erkundung und theologische Herausforderung, „Theologische Revue“ 103 (2007), Nr. 3, S. 177-186.

Englert R. u.a., Einleitung, [in:] Welche Religionspädagogikist pluralitätsfähig? Kontroversen um einen Leitbegriff, Hg. R. Englert u.a., Freiburg i. Br. 2012, S. 9-11.

Englert R., Religion gibt zu denken. Eine Religionsdidaktikin 19 Lehrstücken, München 2013.

Englert R. u.a., Innenansichten des Religionsunterrichts. Fallbeispiele, Analysen, Konsequenzen, München 2014. 
Gabriel K., Pluralisierung und Individualisierung in Gesellschaft, Religion und Kirche, [in:] Christliche Identität in pluraler Gesellschaft, Hg. H. Münk, M. Durst, Freiburg 2005, S. 21-53.

Gärtner C., Religionsunterricht - ein Auslaufmodell? Begründungen und Grundlagen religiöser Bildung in der Schule, Paderborn 2015.

Graf F. W., Die Wiederkehr der Götter. Religion in der modernen Kultur, München 2004.

Grümme B., Bildungsgerechtigkeit. Eine religionspädagogische Herausforderung, Stuttgart 2014.

Grümme B., Öffentliche Religionspädagogik. Religiöse Bildung in pluralen Lebenswelten, Stuttgart 2015.

Grümme B., Altmeyer S., Gerechtigkeit durch religiöse Bildung, „, Theologisch-Praktische Quartalsschrift" 3 (2014), S. 314-324.

Habermas, J., Zwischen Naturalismus und Religion. Philosophische Aufsätze, Frankfurt a. M. 2005.

Hilger G., Heilig ist mir... Texte von Jugendlichen, „Katechetische Blätter 124 (1999), S. 411-412.

Höhn, H.-J., Postsäkular. Gesellschaft im Umbruch - Religion im Wandel, Paderborn 2007.

Zwischen Religion und Religiosität. Ungebundene Religionskulturen in Religionsunterricht und kirchlicher Jugendarbeit - Erkundungen und Praxis, Hg. U. Kropac, U. Meier, K. König, Regensburg 2015.

Lütze F. M., Religiöse Bildung im Hauptschulbildungsgang, „,Theo-Web. Zeitschrift für Religionspädagogik“ 10 (2011), S. 55-79.

Lütze F. M., Religionslehrer/in an Hauptschulen werden. Überlegungen zu schulformspezifischen Akzenten im theologischen Studium, „Theo-Web. Zeitschrift für Religionspädagogik“ 10 (2011), S. 85-97.

Lütze F. M., Religionsunterricht im Hauptschulbildungsgang. Konzeptionelle Grundlagen einer Religionsdidaktik für den Pflichtschulbereich der Sekundarstufe I, Leipzig 2011, S. 68-92.

Pemsel-Maier S., Schambeck M., Keine Angst vor Inhalten! Systematisch-theologische Themen religionsdidaktisch erschließen, Freiburg i. Br. 2015.

Pollack, D., Religion und Moderne. Versuch einer Bestimmung ihres Verhältnisses, [in:] Gottesrede in postsäkularer Kultur, Hg. P. Walter, Essen 2007, S. 19-52.

Porzelt B., Jugendliche Intensiverfahrungen. Qualitativ-empirischer Zugang und religionspädagogische Relevanz, Graz 1999.

Porzelt B., Grundlegung religiöses Lernen, Stuttgart 2009.

Ratzinger J., Die Krise der Katechese und ihre Überwindung. Rede in Frankreich, Einsiedeln 1983.

Riesebrodt M., Die Rückkehr der Religionen: Fundamentalismus und der „Kampf der Kulturen“, München 2000.

Ritter W., Leid und Gott - didaktisch reflektiert, „Religionspädagogische Beiträge“ 59 (2007), S. 3-16.

Schweitzer F., Interreligiöse Bildung, Religiöse Vielfalt als religionspädagogische Herausforderung und Chance, Gütersloh 2014. 
Sellmann M., Jugendliche Religiosität als Sicherungs-und Distinktionsstrategie im sozialen Raum, [in:] Jugend, Religion, Religiosität. Resultate, Probleme und Perspektiven der aktuellen Religiositätsforschung, Hg. U. Kropac, U. Meier, K. König, Regensburg 2012, S. 24-55.

Sellmann M., Zuhören Austauschen Vorschlagen Entdeckungen pastoraltheologischer Milieuforschung, Würzburg 2012.

Shell Deutschland Holding Hg., Jugend 2015. Eine pragmatische Generation im Aufbruch, Hamburg 2015.

Trautmann M., Wischer B., Heterogenität in der Schule, Eine kritische Einführung, Wiesbaden 2011.

Vieregge D., Religiosität in der Lebenswelt sozial benachteiligter Jugendlicher. Eine empirische Studie, Münster 2013.

Walgenbach K., Heterogenität-Intersektionalität -Diversity in der Erziehungswissenschaft, Opladen-Toronto 2014.

Ziebertz H. G., Grenzen des Säkularisierungstheorems, [in:] Entwurf einer pluralitätsfähigen Religionspädagogik, Hg. F. Schweitzer u.a., Freiburg i. Br. 2002, S. 51-74.

Ziebertz H. G., Gesellschaftliche und jugendsoziologische Herausforderungen für die Religionsdidaktik, [in:] Religionsdidaktik. Ein Leitfaden für Studium, Ausbildung und Beruf, Hg. G. Hilger, München 2010, S. 76-105. 